\title{
Análisis de la tasa de detección mediante ecografía de fe- tos con crecimiento intrauterino restringido y pequeño para la edad gestacional
}

\author{
Mariam Abulhaj Martínez, Sara Martínez Chica, Isabel Rodríguez García, Rosario María \\ Redondo Aguilar, María Jesús Teva García.
}

Unidad de Gestión Clínica de Obstetricia y Ginecología. Hospital Universitario San Cecilio, Granada, España.

\section{RESUMEN}

Objetivo: Analizar la tasa de detección de fetos pequeños para la edad gestacional (PEG) y crecimiento intrauterino restringido (CIR) mediante exploración ecográfica. Método: Estudio retrospectivo de casos y controles. Recién nacidos (RN) de gestaciones únicas a término (entre la semana 37-42) en el Hospital Universitario San Cecilio entre 2009 y 2010. Grupo casos: $98 \mathrm{RN}$ a término con peso al nacer $<2500 \mathrm{~g}$, con peso fetal estimado (PFE) por ecografía en el III trimestre. Grupo control: 100 RN durante el mismo período con peso al nacer entre 3000-4000 g, con PFE por ecografía en el III trimestre obtenido de forma aleatoria. Resultados: La tasa de detección de CIR tipo I fue de un $11,1 \%$ con una incidencia del $0,1 \%$ y la tasa de detección de pequeños para edad gestacional fue de un $56,5 \%$ con una incidencia del $2 \%$ respecto al total de RN. Conclusión: En nuestra experiencia la EPF mediante ecografía en fetos con peso bajo el p10 tiene un error de $6 \%$ respecto al peso neonatal, esto permite una adecuada identificación de fetos con PEG y CIR.

\section{PALABRAS CLAVE: Crecimiento intrauterino restringido, pequeño para la edad gestacional}

\section{SUMMARY}

Objective: To analyze the detection rate of small for gestational age (SGA) fetuses and intrauterine growth restriction (IUGR) fetuses by ultrasound exploration. Methods: Cases and controls retrospective study. Material: Newborn (NB) of singleton pregnancies at term (between 37 and 42 weeks) at the Hospital Universitario San Cecilio between 2009 and 2010 both inclusive. Cases group: 98 at term NB with a birth weight $<2500 \mathrm{~g}$; and an estimated fetal weight (EFW) by ultrasound in the 3rd trimester. Controls Group: 100 NB during the same period of time with birth weight between $3000-4000 \mathrm{~g}$, with EFW by ultrasound in the 3rd trimester randomly extracted. Results: The detection rate of small for gestational age infants born was $56.5 \%$ with $2 \%$ of incidence and the detection rate of intrauterine growth restriction type I was $11.1 \%$ with $0.1 \%$ of incidence within the total number of newborns in the period from 2009 to 2010. Conclusion: In our experience, EPF ultrasound in fetuses with weight below the 10th percentile has an error of $6 \%$ compared to birth weight, this allows proper identification of fetuses with SGA and IUGR.

KEY WORDS: Fetal growth restriction, small for gestational age 


\section{INTRODUCCIÓN}

Las alteraciones del crecimiento intrauterino son una situación frecuente en obstetricia. Se considera un feto pequeño para edad gestacional (PEG) el que tiene un peso estimado inferior al p10. Se define el crecimiento intrauterino restringido (CIR) como: a) la presencia de un peso fetal estimado (PFE) inferior al p3 o b) un peso estimado entre p3 y p10 con alteración del flujo cerebro-placentario o de las arterias uterinas. Se produce una limitación del potencial de crecimiento intrínseco del feto debido a factores uteroplacentarios. La dificultad radica en que no podemos conocer cuál es el potencial de crecimiento intrínseco de cada feto, por ello nos tenemos que basar en tablas de peso adecuadas a nuestra población y los percentiles de normalidad. Por lo tanto, el estudio Doppler es esencial para diferenciar entre los fetos que presentan un CIR de los PEG. Sin embargo, también es importante discriminar aquéllos que presentan alguna patología asociada (PEG anormales) (1). Debemos tener en cuenta que el $70 \%$ de los fetos con peso estimado por debajo del percentil 10 son simplemente pequeños por factores constitucionales tales como: sexo femenino, raza étnica, índice ponderal materno, paridad. Estos fetos no tienen aumentada la morbimortalidad (2).

En definitiva, los fetos con peso estimado $<$ p10 se clasifican en: a) PEG anormal: si PFE < p10 y anomalía estructural, genética o infecciosa. b) PEG normal: si PFE < p10 y lo demás normal. c) CIR:

Tipo I: PFE < p3 + índice cerebro-placentario (ICP) normal. Índice de pulsatilidad (IP) arterias uterinas (AUt) normal.

Tipo II: PFE < p10 + ICP < p5 ó IP AUt alterado. Tipo III: PFE < p10 + flujo diastólico ausente en AUt. Tipo IV: PFE < p10 + ICP < p5 + arteria cerebral media $(A C M)<p 5$.

Tipo V: PFE < p10 + 1 ó más criterios de gravedad: perfil biofísico alterado, registro frecuencia cardiaca fetal alterado (sin variabilidad o con desaceleraciones), flujo diastólico reverso en AUt, IP ductus venoso > p95 y/o pulsaciones en la vena umbilical. Los factores de riesgo son múltiples y no siempre bien definidos; entre ellos los trastornos hipertensivos del embarazo están presentes en 1/3 de las alteraciones del crecimiento fetal (3). En este sentido el flujo Doppler de las arterias uterinas en el I y II trimestre tiene un papel muy importante en la predicción de preeclampsia (4).

La detección de los fetos con déficit del crecimiento es de gran importancia ya que el riesgo de resultados perinatales adversos es hasta cuatro veces mayor en aquellos no identificados antena- talmente. La ecografía es el gold standard para la estimación biométrica fetal $(1,5)$. Esto requiere tres pasos fundamentales: correcta datación de la edad gestacional, estimación antenatal del crecimiento y valoración del crecimiento fetal con estándares adecuados.

Se suelen utilizar las biometrías fetales medidas por ecografía pero esta estrategia ha mostrado un alto número de falsos positivos. Sin embargo, las fórmulas de cálculo de peso fetal a partir de los datos biométricos tienen una alta validez, especialmente las que incorporan medidas cefálicas, abdominales y de las extremidades (6). Aunque no debemos olvidar que para todas las fórmulas consideradas, el error entre el peso real y el estimado no es inferior al 7,5-10\% (7).

El objetivo del estudio es analizar la tasa de detección de fetos PEG y con CIR mediante exploración ecográfica en la Unidad de Gestión Clínica de Obstetricia y Ginecología (UGC) del Hospital Universitario San Cecilio durante los años 2009 y 2010.

\section{MATERIAL Y MÉTODOS}

Tipo de estudio: Estudio retrospectivo de casos y controles.

Material: Recién nacidos $(\mathrm{RN})$ de gestaciones únicas a término (entre la semana 37 y 42) en el Hospital Universitario San Cecilio (HUSC) entre 2009 y 2010 ambos inclusive.

Grupo casos: $98 \mathrm{RN}$ a término con peso al nacer < $2500 \mathrm{~g}$; con PFE por ecografía en el III trimestre. Consideramos pérdidas un total de $39 \mathrm{RN}$ con peso $<2500 \mathrm{~g}$ en los que no queda constancia de PFE. Grupo controles: $100 \mathrm{RN}$ durante el mismo período de tiempo con peso al nacer entre 3000-4000 g, con PFE por ecografía en el III trimestre. Este grupo se extrae del total de los recién nacidos entre 3000 y $4000 \mathrm{~g}$ entre el año 2009-2010 de forma aleatoria.

Los pesos fetales son estimados mediante exploración ecográfica a partir de las medidas del diámetro biparietal, circunferencia abdominal y longitud del fémur. Se emplean para el cálculo del PFE las tablas de Hadlock predeterminadas en los ecógrafos de nuestra unidad. Los datos fueron obtenidos mediante la revisión de las historias clínicas recogidas en la base electrónica intrahospitalaria. Se excluyeron gestaciones gemelares a término con recién nacidos con peso $<2.500 \mathrm{~g}$, así mismo, no se han incluido gestaciones $<37$ semanas, ni gestaciones > 42 semanas. Hemos excluido del estudio (total de la muestra) un total de 39 pérdidas por inexistencia de PFE en nuestra base de datos. 
Método: El análisis descriptivo de los datos abarca tanto en el grupo caso como en el control las siguientes variables: edad materna, fórmula obstétrica, antecedentes personales maternos, terminación de la gestación. Se realiza test de comparación de 2 medias para muestras independientes (Test de Student), con el objetivo de comparar el peso del recién nacido con el peso fetal estimado en ecografía del III trimestre. Se analizan los datos mediante el paquete estadístico SPSS 15.0. Todos los datos analizados y descritos se han obtenido de la base de datos del Hospital Universitario San Cecilio de Granada, el cual atiende al área Sur de Granada y es centro de referencia para otros hospitales de menor nivel asistencial.

\section{RESULTADOS}

Durante los años 2009 y 2010 hubo un total de 5576 partos en nuestro hospital. La incidencia de recién nacidos $P E G$ fue de un $2 \%$ respecto al total de recién nacidos en el período de 2009 a 2010. La incidencia de recién nacidos con CIR tipo I fue de un $0,1 \%$ respecto al total de $\mathrm{RN}$ durante el período de estudio.

En el grupo de recién nacidos con peso inferior a $2500 \mathrm{~g}$, encontramos que tan sólo el $1 \%$ de los casos fueron embarazos cronológicamente prolongados (41-42 semanas) mientras que en el grupo control fueron $24,7 \%$. La terminación del parto en el grupo de recién nacidos de peso $<2500 \mathrm{~g}$ fue de forma espontánea en el $63,3 \%$ de los casos, acabando en cesárea el $29,6 \%$, y partos instrumentales el $7,1 \%$ de los casos. Por el contrario en el grupo control encontramos un total de un $76,3 \%$ de partos espontáneos, $17,5 \%$ de cesáreas y un $6,2 \%$ de partos instrumentales.

La media de edad materna fue de 31,78 años (DS $\pm 5,54$ años) y la mayoría de las gestantes eran nulíparas en el grupo de casos $(59,2 \%)$. En el grupo control la muestra se considera homogénea en cuanto a edad materna y fórmula obstétrica.

Entre los antecedentes personales maternos en el grupo de recién nacidos con peso $<$ p10 para la edad gestacional, los más destacados fueron: estados hipertensivos del embarazo (16,3\%) (hipertensión inducida por el embarazo, hipertensión crónica y preeclampsia), oligoamnios diagnosticado prenatalmente $(10 \%)$, diabetes gestacional $(3,1 \%)$, alteraciones tiroideas $(3,1 \%)$, obesidad $(2,9 \%)$ y arteria umbilical única diagnosticada prenatalmente $(2 \%)$.

En el grupo control evidenciamos un $7,2 \%$ de estados hipertensivos, además, no se evidenció ningún caso de arteria umbilical única ni oligoamnios, alteraciones tiroideas en un $2,1 \% ; 1 \%$ de dia- betes gestacional, sin encontrar ningún caso de diabetes pregestacional.

La tasa de detección de CIR tipo I (peso inferior al percentil 3, sin signos de redistribución hemodinámica) fue de un $11,1 \%$, la tasa de detección de pequeños para la edad gestacional (PEG; peso entre los percentiles 3 al 10 poblacional sin alteraciones hemodinámicas; con Doppler de arteria umbilical y arteria cerebral media normales) fue de $56,5 \%$.

La media de pesos fetales estimados en el grupo control fue de $3204,8 \mathrm{~g}$ (DS $\pm 299,9 \mathrm{~g}$ ), mientras que en el grupo de los casos, la media fue de 2441 $g(D S \pm 370,4 \mathrm{~g})$.

Al realizar el test de comparación de 2 medias para muestras independientes (Test de Student), se calcula el error entre el peso fetal estimado y el peso al nacer. En el grupo de controles la media diferencial se estima en $206,5 \mathrm{~g}$, mientras que en los recién nacidos del grupo de casos la diferencia encontrada es de 143,6 g. Al comparar el error en la estimación ecográfica en ambos grupos, la diferencia es significativa ( $<<0,001$ con IC95\%: 250,9 $449,4 \mathrm{~g})$.

\section{DISCUSIÓN}

Durante los años 2009 y 2010 hubo un total de 5576 partos en el HUSC siendo la incidencia de RN con peso < p10 para su edad gestacional de 2,1\%. Las revisiones sistemáticas recogidas en la literatura hablan de una incidencia que oscila entre el 4 y el $15 \%$ (8). La mayor morbi-mortalidad que presentan estos $\mathrm{RN}$ supone un importante impacto en los servicios destinados a su atención, y esto hace fundamental su detección antenatal.

Un primer paso para mejorar el diagnóstico antenatal es detectar la presencia de factores de riesgo de retraso del crecimiento fetal. Entre nuestra población hasta un $16,3 \%$ de las gestaciones con RN con peso < p10 presentaron trastornos hipertensivos. Estos representan el principal factor de riesgo para el desarrollo de CIR ya que están presentes en 1/3 de las alteraciones del crecimiento fetal (3). Nuestra Unidad de Gestión Clínica (UGC) ha implantado durante el último año un programa de cribado de preeclampsia que se realiza en la consulta de I trimestre mediante el análisis del flujo Doppler de ambas arterias uterinas y la toma de tensión arterial en ambos brazos. Se realiza a todas aquellas gestantes que presentan riesgo elevado de preeclampsia. Si el resultado del cribado es de alto riesgo se inicia tratamiento profiláctico con AAS $100 \mathrm{mg} /$ día que se mantiene hasta la semana 36 de gestación, y control Doppler de las arterias uterinas en las semanas 
16, 20 y 24. Este protocolo ha permitido mejorar la detección precoz de trastornos hipertensivos en las embarazadas de riesgo, mejorar la detección de fetos pequeños asociados a dichos trastornos así como su seguimiento, y finalmente mejorar los resultados perinatales.

La correcta datación de la gestación es un paso previo fundamental para mejorar el diagnóstico ecográfico de las alteraciones en el crecimiento fetal. En este sentido, nuestra UGC ha implantado una consulta para cribado de cromosomopatías de I trimestre que es de ámbito universal en nuestra población. Siguiendo las últimas recomendaciones, se realiza la datación de la gestación en función de la longitud céfalo-nalgas (LCN) en esa visita de I trimestre.

Estas nuevas medidas, tanto el cribado de preeclampsia en I trimestre como la datación mediante LCN en el I trimestre, han sido de reciente implantación en nustra UGC. Por tanto, aún es pronto para valorar el impacto real de las mismas a largo plazo, aunque los resultados iniciales son muy positivos.

Menos del $1 \%$ de los fetos con peso < p10 nacieron más allá de la semana 41. Probablemente este porcentaje recoja aquéllos que fueron catalogados ecográficamente como falsos negativos con PFE normal para edad gestacional y se les dejó evolucionar hasta el embarazo cronológicamente prolongado. El resto nacieron antes de la semana 40 , tal y como indican los protocolos de manejo de fetos con CIR o PEG, consiguiendo una tasa de partos espontáneos del 63,3\%.

Diferentes estudios han intentado determinar cuál es el mejor método para realizar la estimación del peso fetal mediante ecografía. La mayoría coinciden en que el empleo de las tablas desarrolladas por Hadlock es el que permite una mejor aproximación al peso real. Nuestros ecográfos tienen predeterminadas las tablas Hadlock para el cálculo del peso fetal a partir de la medida del diámetro biparietal, circunferencia abdominal y longitud del fémur. Por supuesto, es fundamental realizar estas medidas siguiendo los estándares de calidad establecidos (8). Un campo de mejora en este sentido puede ser entrenar las habilidades de los facultativos responsables de la ecografía del III trimestre de la gestación, así como que la EPF sea realizada por dos facultativos distintos antes de tomar una decisión en cuanto al manejo o el momento de finalización.

Las publicaciones y revisiones a este respecto muestran que para todas las fórmulas de cálculo del peso fetal consideradas, el error entre el peso real y el estimado no es inferior a $7,5-10 \%(7,9)$. Nuestros resultados muestran una tasa de error en la detección de fetos con peso < p10 del 6\% (143,6 g), mejorando las tasas que reflejan otros estudios a nivel mundial (10). En nuestro estudio encontramos una tasa de detección de fetos PEG del 56,5\% y de CIR del $11,1 \%$, ambas cifras también superiores a las recogidas en distintos estudios internacionales $(7,10)$.

\section{CONCLUSIÓN}

Nuestra experiencia muestra que la EPF mediante ecografía en fetos con peso bajo el p10 tiene un error de $6 \%$ respecto al peso neonatal, esto permite una adecuada identificación de fetos con PEG y CIR.

\section{REFERENCIAS}

1. Gratacós E, Gómez R, Romero R, Cabero L. Alteraciones del crecimiento fetal. En: Medicina Fetal. Editorial Médica Panamericana, SA, 2007; Capítulo 71:639-48.

2. Crecimiento Intrauterino Restringido. Protocolo SEGO 2007. En: http://www.prosego.com/index.php?option= content\&task=view\&id $=539 \&$ Itemid $=141$

3. Audibert F, Benchimol Y, Benattar C, Champagne C, Frydman R. Prediction of preeclampsia or intrauterine growth restriction by second trimester serum screening and uterine Doppler velocimetry. Fetal Diagn Ther 2005;20:48-53.

4. Carbillon L, Lynch AM, Cioffi-Ragan D, Hobbins JC, Schultz LK, Malone FD, D'Alton ME. First trimester uterine artery Doppler abnormalities predict subsequent intrauterine growth restriction. Am J Obstet Gynecol 2006;195:1208-12.

5. Peleg D, Kennedy M, Hunter S. Intrauterine growth restriction: identification and management. Am Fam Phy 2000;58:453-60.

6. Gardosi J. Fetal growth: towards an international standard. Ultrasound Obstet Gynecol 2005; 26:112-4.

7. Mandruzzato G, Maso G. Crecimiento fetal. Crecimiento.indd. 2009;7:118-20. Hallado en: http:// www.iguana2007.com/amolca-jul-cliente/pdf/pagina-118-3120.pdf

8. Fiestas C, Valera D, Palacios J, Gonzáles L, Bardales $\mathrm{B}$, Cisneros J. Comparación de dos fórmulas para calcular el peso fetal ecográfico vs peso al nacer. Ginecol. obstet. 2003;49: 214-18. Hallado en: http://sisbib. unmsm.edu.pe/bvrevistas/ginecologia/vol49_n4/a03. $\mathrm{htm}$

9. Robert Resnik, MD.Fetal growth restriction: Evaluation and management. Up to date. Hallado en: http:// www.uptodate.com/contents/fetal-growth-restrictionevaluation-and-management?source=search_result \&search=Intrauterine+growth+restriction\&selectedTit le $=1 \sim 150$

10. Sanín Blair JE, Gómez Díaz J, Ramírez J, Mejía CA, Medina A, Vélez J. Diagnóstico y seguimiento del feto con restricción del crecimiento intrauterino y del feto pequeño para edad gestacional. Consenso Colombiano. Rev Colomb Obstet Ginecol 2009;60:247-61. 